\title{
Abstract \\ Analysis of maternal and child health care status in Anuradhapura District for 5 years period since 2010 to 2015
}

Weerakoon $\mathrm{H}^{1 *}$

${ }^{1}$ PDHS Office, Anuradhapura

\begin{abstract}
Background

In Sri Lanka, The health indications show steady improvement over recent decades particularly in maternal and child health $(\mathrm{MCH})$ care. Pregnancy and childbirth have a huge impact on the physical, mental, emotional and socio economic health of women and their families. Objectives were to create awareness among care givers on $\mathrm{MCH}$ status, to analyze gap in the $\mathrm{MCH}$ services and to predict $\mathrm{MCH}$ trends and estimating future needs of community and fix suitable targets for achievements.

\section{Methods}

Data were recorded from maternal and child health returns (H509) for a 5 year period and analyzed.

\section{Results}

During this 5 year period each year had nearly 15,000 births with increasing low birth rate, recent increment of home delivery rate and reduction of still birth rate. There is reduction of teenage pregnancy rate, increase rate of lower segment cesarean section, increase antenatal morbidity rate and reduce maternal body mass index rate. During this 5 year period there is a fluctuation in maternal mortality rate, infant mortality rate and abortion rate. Concerning nutrition status, children of 2-5 years and children under 5 year the underweight rates reduced yearly but infant and children of 1-2 years shows fluctuations. Concerning family planning current users of family planning method show fluctuations and usage of oral contraceptive pills, intrauterine devices and implants increased and unmet needs reduced.

Conclusions
\end{abstract}

Need to take measures to address nutrition status and reduce antenatal morbidities.

Key words: Matternal and child health; Anuradhapura District; Five years period

Copyright: $\odot 2015$ Weerakoon $\mathrm{H}$ et al. This is an open access article distributed under the Creative Commons Attribution License, which permits unrestricted use, distribution, and reproduction in any medium, provided the original work is properly cited.

*Correspondence : hemaweerakoon68@gmail.com

Cite this abstract as: Weerakoon $\mathrm{H}$. Analysis of maternal and child health $(\mathrm{MCH})$ care status in Anuradhapura District for 5 years period since 2010 to 2015. Anuradhapura Medical Journal 2015;9 (2Supp):S35.

DOI: http://dx.doi.org/10.4038/amj.v9i2Supp.7584 
